\title{
DRI: SÍNTESE COMENTADA DAS NOVAS PROPOSTAS SOBRE RECOMENDAÇÕES NUTRICIONAIS PARA ANTIOXIDANTES
}

\author{
DRI: COMMENTED NOTE OF THE NEW NUTRITIONAL \\ RECOMMENDATIONS FOR DIETARY ANTIOXIDANTS
}

\author{
Jaime AMAYA-FARFAN' \\ Semíramis Martins Álvares DOMENE² \\ Renata Maria PADOVANI'
}

\begin{abstract}
RESUMO
Discute-se o conjunto dos recentes relatórios do Institute of Medicine e Food and Nutrition Board da US National Academy of Sciences, publicados entre 1998 e 2000, dando-se particular ênfase ao livro das propostas de Ingestão Alimentar de Referência para os denominados antioxidantes alimentares. $O$ conceito das DRI engloba os já conhecidos valores de Recomendações de Cotas ou Doses Alimentares (RDA), os novos valores de Ingestão Adequada, Ingestão Máxima Tolerável e as Estimativas Médias dos Requerimentos. O termo RDA continua em uso, e é reservado para a recomendação mais apurada de um determinado nutriente, da qual se tenha informação. Na tabela da Ingestão Alimentar de Referência, encontram-se as Recomendações de Doses Alimentares atualizadas e complementadas com valores de Ingestão Recomendada de Nutrientes canadenses, ou completadas com valores de Ingestão Adequada. Valores de Ingestão Máxima Tolerável para os antioxidantes, exceto os carotenóides, são fornecidos em separado.
\end{abstract}

Termos de indexação: nutrientes, ingestão, recomendações, dieta, planejamento alimentar.

\begin{abstract}
This is a brief, commented review of the 1998-2000 reports of the Institute of Medicine/Food and Nutrition Board, of the US National Academy of Sciences, particularly that concerning the recommendations for dietary antioxidants. Dietary Reference Intakes (DRI) include Recommend Dietary Allowance (RDA), the new Adequate Intakes and the concepts of Upper Tolerable Intake Levels and Estimated Average Requirements. The term RDA therefore has not disappeared and continues to represent the most refined value known for a numerical recommendation. The Dietary Reference Intakes table is an ensemble of Recommended Dietary Allowance that were either updated, complemented with the Canadian Recommended Nutrient Intake, or completed with Adequate Intake values. Upper Tolerable Intake Leved for the antioxidants, except for the carotenoids, have been proposed in a separate table.
\end{abstract}

Index terms: nutrients, ingestion, recommendations, diet, food planning.

\footnotetext{
(1) Departamento de Planejamento Alimentar e Nutrição, Faculdade de Engenharia de Alimentos, Universidade Estadual de Campinas.

(2) Curso de Nutrição, Faculdade de Ciências Médicas, PUC-Campinas. Av. John Boyd Dunlop, s/n., Bloco A, Jd Ipaussurama, 13059-900, Campinas, SP, Brasil. Correspondência para/Correspondence to: S.M.A. DOMENE.
} 


\section{INTRODUÇÃO}

O volume e diversificação da informação química, nutricional e toxicológica acumulada por mais de meio século sobre a ingestão alimentar de nutrientes e o que é mais seguro para o homem, fez com que a simples atualização das clássicas Recommended Dietary Allowance (RDA) se tornasse inadequada.

No final de 1999, início de 2000, a Food and Nutrition Board, da US National Academy of Sciences, publicou a versão em papel do último dos três primeiros volumes sobre as novas Dietary Reference Intakes (DRI), ou "Ingestão Alimentar de Referência", para três grupos de nutrientes relacionados entre si, a saber: 1) DRI para cálcio, fósforo, magnésio, vitamina D e fluoreto; 2) DRI para tiamina, riboflavina, niacina, vitamina $B_{6}$, folato, vitamina $B_{12}$, pantotenato, biotina e colina e, 3) DRI para os antioxidantes vitamina $C$, vitamina $E$, selênio e carotenóides. Afastando-se do esquema clássico, a Comissão optou por relatar os nutrientes por grupos correlatos, em lugar dos já tradicionais grupos de "vitaminas hidrossolúvies", "vitaminas lipossolúveis", "elementos traço", etc. Dessa forma, o leitor encontrará o material mais logicamente integrado e mais interessante (US National Academy..., 2000).

Este trabalho é uma revisão abreviada do conceito todo de DRI proposto pela Comissão para os já reconhecidos antioxidantes alimentares, quais sejam: a vitamina $C$, vitamina E e o selênio, assim como se discutem os efeitos benéficos e adversos do consumo destes, do $\beta$-caroteno e outros carotenóides ( $\alpha$-caroteno, $\beta$-criptoxantina, licopeno e zeaxantina), mas sem emitir recomendações quantitativas sobre os cinco últimos. É positivo observar que estas substâncias são chamadas às vezes de "nutrientes" e fica claro que a literatura existente, embora insuficiente para permitir a determinação de recomendações numéricas sobre seu consumo, aponta a presença de ações biológicas variadas, nem sempre relacionadas com a ação antioxidante.

As definições usuais são:

- RDA (Recommended Dietary Allowance, ou "Recomendações de Doses ou Cotas Alimentares"): é o nível de consumo alimentar de cada nutriente, suficiente para satisfazer os requerimentos de quase todo indivíduo saudável (entre 97 e 98\%), compreendido num determinado grupo, por gênero, faixa etária e estágio de vida (o termo continua em uso e denota a recomendação mais apurada à qual se possa chegar).

- Al (Adequate Intake, ou "Ingestão Adequada"): um valor de consumo recomendável, baseado em levantamentos, determinações ou aproximações de dados experimentais, ou ainda de estimativas de ingestão de nutrientes para grupo(s) de pessoas sadias, e que se considera adequado. É usado quando a RDA não pode ser determinada.
- UL (Tolerable Upper Intake Level, ou "Limite de Ingestão Máxima Tolerável): o mais alto nível de ingestão de um nutriente que não causará efeitos adversos à saúde da maioria das pessoas. Acima do $U L$, o risco de efeitos adversos aumenta sensivelmente.

- EAR (Estimated Average Requirement ou "Estimativa do Requerimento Médio"): valor (mediana) suficiente para garantir o requerimento de $50 \%$ dos indivíduos sadios compreendidos num determinado estágio da vida (prefere-se o termo 'estágio-de-vida' ao invés de 'faixa etária').

$D R I$ "são valores numéricos estimados de consumo de nutrientes para uso no planejamento e avaliação de dietas para pessoas aparentemente saudáveis". O novo conjunto de valores compreende as RDA (ou algumas RNI do Canadá) e as AI. Embora os conceitos (e valores) de UL e EAR tenham entrado na elaboração da tabela principal, esses valores não constam dela, pois as EAR são inferiores e os UL muito superiores às RDA. Devido à presença de lacunas nos estudos, foi necessária a avaliação crítica e interpretação da literatura existente em cada Sub-Comitê de especialistas. O Painel de Especialistas em Antioxidantes e Compostos Afins contava com dois Sub-Comitês: o Subcommittee on Upper Reference Levels of Nutrients e o Subcommittee on Interpretation and Uses of Dietary Reference Intakes.

Dentre as principais metas alcançadas pela Comissão estão:

- Elaboração da definição de antioxidante alimentar;

- Conclusão de que as RDA para vitamina E e selênio não deveriam variar em função do gênero ou idade depois dos 14 anos, de certa forma refletindo a inespecificidade dos atuais dados;

- Estabelecimento de que os requerimentos e recomendações em vitamina $\mathrm{E}$ deveriam ser baseados exclusivamente no $\alpha$-tocoferol, devido ao fato de os outros isômeros e os tocotrienóis não serem convertidos para $\alpha$-tocoferol e mal serem reconhecidos pela proteína transferidora do fígado;

- Confirmação de que as RDA em vitamina C para homens são maiores do que para mulheres, apenas devido às diferenças em massa corpórea;

- Estabelecimento dos UL para vitamina C, vitamina E e selênio;

- Recomendação de pesquisas de intervenção em grande escala sobre os potenciais efeitos preventivos de doenças pelos antioxidantes, inclusive o $\beta$-caroteno e os outros carotenóides.

\section{Como se chegou às RDA?}

Para melhor entender o significado de todos estes termos, é conveniente lembrar que uma 
recomendação 'RDA' é obtida do requerimento médio conhecido de um certo nutriente. Como a recomendação certamente será maior do que o requerimento fisiológico (eliminando assim as diferenças oriundas de variações genéticas, biodisponibilidade, etc.), a questão se reduziu a escolher o tamanho da margem de segurança a ser utilizada ao se lançar o valor de RDA. A média do requerimento é a chamada de 'EAR', ou estimativa de requerimento médio.

O procedimento representa um passo além do já descrito em edições anteriores. Para o estabelecimento de uma RDA foi requisito estabelecer primeiro a EAR, para a qual se deveria contar de antemão com algum critério do que seria um consumo adequado. Dentre os critérios usados, foi dada ênfase aos parâmetros de saúde, como a redução do risco de doenças, abordagem esta que, embora timidamente colocada nas RDA de 1989, pode ser considerada inovadora, pois diminuiu o peso dado ao fator crescimento.

Quando os desvios-padrão (DP) de uma EAR eram conhecidos e a distribuição estatística era simétrica, foi possível então obter a RDA pela expressão: $\mathrm{RDA}=\mathrm{EAR}+2 \mathrm{DP}_{\mathrm{EAR}^{\prime}}$ como se ilustrava antigamente com a proteína. Isto é, ao acrescentar duas vezes o desvio-padrão à média, estende-se a cobertura, de 50 para algo em torno de $97,5 \%$ da população. Nos casos em que não foi possível calcular o DP, optou-se por utilizar um Coeficiente de Variação (CV) geral de 10,0\%, obtendo-se então a expressão: RDA $=1,2 \times$ EAR. Já nos casos em que embora conhecendo-se a EAR, a distribuição para um grupo não era simétrica, outros mecanismos de estimativa foram empregados.

$\mathrm{Na}$ inexistência de dados suficientes para se determinar uma EAR, optou-se então por fornecer simplesmente os dados de ingestão adequada. Isto pode ser visto, por exemplo, na Tabela 2 (valores com *) para o grupo de lactentes de zero a 12 meses, independente do nutriente; ou para vitamina D, fluoreto, ácido pantotênico, biotina e colina, independente da idade do indivíduo.

Para a maioria dos nutrientes, a Al para lactentes (4 a 6 meses) foi baseada em médias de ingestão diária (de bebês aparentemente saudáveis, a termo e sob aleitamento materno exclusivo, como é recomendado).

Tanto as RDA quanto as $\mathrm{Al}$ podem ser usadas na avaliação de dietas de indivíduos, a diferença sendo que as Al, são via de regra quantidades maiores; isto para minimizar a probabilidade de que muitos indivíduos não fiquem cobertos. De toda forma, é lembrado que casos com requerimentos especiais devem ser avaliados por profissionais qualificados.

A RDA para um nutriente deve ser empregada para orientar a ingestão dietética média de indivíduos saudáveis, e pretende ser parâmetro para a avaliação de dietas de indivíduos ou grupos, ou para o planejamento dietético destes, considerando-se períodos de uma semana.
O novo parâmetro denominado Limite de Ingestão Máxima Tolerável (UL) é o nível mais alto de ingestão do nutriente que pode ser tolerado sem apresentar efeitos adversos 'observáveis' para quase todos os indivíduos da população em geral. Um valor de UL não pode ser entendido como recomendação permissível de ingestão dietética. O relatório ressalta que ainda não foram estabelecidos os efeitos benéficos para indivíduos saudáveis que consomem nutrientes acima da RDA ou AI. Não obstante, devemos lembrar que permanece uma certa dificuldade em definir o que é um indivíduo saudável, especialmente para idades superiores aos 70 anos.

Contrário ao que se desejava, o sub-comitê não só deixou de emitir recomendações para os carotenóides, como não encontrou dados suficientes para estabelecer UL para o $\beta$-caroteno e outros carotenóides. Isto não significa, entretanto, que esses nutrientes estejam liberados para consumo em qualquer quantidade, ou que não haja efeitos adversos em potencial associados às altas taxas de ingestão, particularmente para o $\beta$-caroteno em fumantes. Futuramente, talvez chegue-se a um UL próximo dos $10 \mathrm{mg} /$ dia. Importante é frisar também que os UL se referem à ingestão diária de forma crônica.

\section{Funções biológicas e indicadores bioquímicos usados como critérios para determinar os requerimentos dos antioxidantes}

Vitamina C: Devido ao seu alto poder redutor, o ácido ascórbico proporciona proteção contra a oxidação descontrolada no meio aquoso da célula. A RDA para mulheres adultas foi estipulada em 75 e, para homens, em $90 \mathrm{mg} /$ dia. Já os fumantes, que têm maior estresse oxidativo, devem aumentar a sua ingestão em 35 mg/dia (Kallner et al., 1981). Nessas doses ainda haverá baixa excreção de metabólitos urinários, indicando que a dosagem não é excessiva.

Vitamina E: Ao que tudo indica, a principal função do $\alpha$-tocoferol é interromper as reações em cadeia (com radicais livres) que ocorrem quando a porção lipídica das células (membranas) entra em peroxidação. O estabelecimento da recomendação de $15 \mathrm{mg} / \mathrm{dia}$ para homens e mulheres (Tabela 2), baseou-se em estudos de deficiência induzida em humanos e ensaios de hemólise in vitro causada pelo peróxido de hidrogênio. $O \alpha$-tocoferol é definido como sendo o isômero $R R R$ - $\alpha$-tocoferol, o único naturalmente presente em alimentos, e os isômeros $2 R$ que são encontrados em produtos fortificados e suplementos (os RRR-, RSR-, RRS-, RSS- $\alpha$-tocoferóis).

Selênio: Este elemento funciona associado às seleno-proteínas, muitas das quais são enzimas que protegem contra a oxidação descontrolada no organismo. Os $55 \mu \mathrm{g} / \mathrm{d}$, para homem ou mulher (Tabela 2), foram recomendados tendo por base a ingestão de selênio que promove atividade máxima para a glutationa peroxidase. 
$\beta$-Caroteno e outros carotenóides provitamínicos A: Por não se considerar suficientes os dados consignados na literatura, não se emitiram DRI nem AI para $\beta$-caroteno, $\beta$-criptoxantina e $\alpha$-caroteno, mesmo na sua atividade provitamínica. Em futuros relatórios, a Comissão espera se pronunciar à respeito das proporções em que estes nutrientes devem participar na dieta. Espera-se também abranger as funções e os carotenóides não provitamínicos.

\section{Critérios e valores propostos para os ULS}

O UL de 2000 mg/dia de vitamina C para adultos, por exemplo (Tabela 1), foram baseadas no efeito adverso da indução de diarréia osmótica, enquanto que os 1000 $\mathrm{mg} /$ dia de $\alpha$-tocoferol suplementar foram fundamentados no efeito adverso do aumento do risco de hemorragias. Já para o selênio o limite foi de $400 \mu \mathrm{g} / \mathrm{dia}$, porque acima dessa ingestão o risco de selenose se torna considerável. O UL para vitamina $C$ e selênio foram baseadas na ingestão de dietas e suplementos. Por sua vez, os UL para vitamina E basearam-se na ingestão de suplementos.

A razão pela qual os UL para $\beta$-caroteno não puderam ser estabelecidos foi a divergência entre alguns estudos, enquanto que para os outros carotenóides os dados confiáveis eram insuficientes. As pesquisas divergentes às quais a Comissão aparentemente se refere são aqueles grandes estudos clínicos de intervenção com cápsulas. Foram três importantes experimentos: 1) O de Linxian (China), de prevenção primária de câncer (Blot et al., 1993), contando com 29584 homens e mulheres
(40-69 anos) em estado de subnutrição. A intervenção foi com dose diária de $15 \mathrm{mg}$ de $\beta$-caroteno, $30 \mathrm{mg}$ de $\alpha$-tocoferol e $50 \mu \mathrm{g}$ de selênio. Os resultados mostraram redução de $21 \%$ nas mortes por câncer estomacal, de 13\% na mortalidade em todos os tipos de câncer e $9 \%$ na mortalidade total. 2) O estudo da Finlândia (ATBC Cancer..., 1994), de prevenção secundária de câncer, contando com 29133 voluntários (homens fumantes "pesados", 50-69 anos, diagnosticados com câncer pulmonar), que recebiam $50 \mathrm{mg}$ de $\alpha$-tocoferol e/ou $20 \mathrm{mg}$ de $\beta$-caroteno. Os resultados mostraram aumento de $50 \%$ nas mortes por derrame hemorrágico no grupo suplementado com vitamina $\mathrm{E}$, aumento de $11 \%$ na mortalidade por isquemia do miocárdio e de $18 \%$ nas mortes por câncer do pulmão no grupo com $\beta$-caroteno. Por sua vez, nenhum efeito atribuível ao tocoferol foi visto no câncer pulmonar. 3) O terceiro, realizado nos EUA, era sobre a prevenção primária de câncer do pulmão e contava com 14254 fumantes "pesados" e 4060 trabalhadores da indústria do asbesto (45-69 anos). Estes consumiam $30 \mathrm{mg}$ de $\beta$-caroteno e 25000 UI de retinol por dia e o resultado mostrou aumento de $28 \%$ na incidência de câncer do pulmão e $17 \%$ na mortalidade geral (Omenn et al., 1996).

\section{Definição de antioxidante alimentar (US NATIONAL..., 2000)}

"Antioxidante alimentar é toda substância na dieta capaz de reduzir significativamente os efeitos adversos produzidos por espécies reativas, como aquelas de oxigênio e nitrogênio, e que possuem função normal no organismo". Nesse sentido, as vitaminas C e E e o selênio (na forma de

Tabela 1. Limites de ingestão máxima tolerável para os antioxidantes.

\begin{tabular}{|c|c|c|c|}
\hline \multirow{2}{*}{ Estágio da vida } & Vitamina C & Vitamina E & Selênio \\
\hline & (mg/dia) & (mg/dia) & (mcg/dia) \\
\hline de 0 a 6 meses & $\mathrm{s} /$ determinar & $\mathrm{s} /$ determinar & 45 \\
\hline de 7 a 12 meses & $\mathrm{s} /$ determinar & $\mathrm{s} /$ determinar & 60 \\
\hline 1 a 3 anos & 400 & 200 & 90 \\
\hline 4 a 8 anos & 650 & 300 & 150 \\
\hline 9 a 13 anos & 1200 & 600 & 280 \\
\hline 14 a 18 anos & 1800 & 800 & 400 \\
\hline 19 a 70 anos & 2000 & 1000 & 400 \\
\hline$>70$ anos & 2000 & 1000 & 400 \\
\hline \multicolumn{4}{|l|}{ na gravidez } \\
\hline$\leq 18$ anos & 1800 & 800 & 400 \\
\hline 19 a 50 anos & 2000 & 1000 & 400 \\
\hline \multicolumn{4}{|l|}{ Lactantes } \\
\hline$\leq 18$ anos & 1800 & 800 & 400 \\
\hline 19 a 50 anos & 2000 & 1000 & 400 \\
\hline
\end{tabular}

Fonte: Institute of Medicine/Food Nutrition Board, US National Academy of Sciences. DRIs for vitamin C, vitamin E and selenium. Washington DC: National Academy Press, 2000 (com autorização). 
seleno-metionina e seleno-cisteína) cumprem a definição, enquanto que $\beta$-caroteno e os outros carotenóides foram considerados como não se ajustando à definição, mas que influenciam as reações bioquímicas do sistema oxidativo. A cautela da Comissão aparentemente se justifica pela forma variável com que certos carotenóides agem; por exemplo, a característica do $\beta$-caroteno de agir como antioxidante quando em baixas concentrações, e como pró-oxidante, quando em altas concentrações.

\section{Relação entre o estresse oxidativo e doenças crônicas}

Uma das características louváveis da nova versão foi a inclusão de considerável volume de informação indicando que o excesso de espécies reativas de oxigênio e nitrogênio ocasionam danos às células normais e, portanto, causam disfunção celular e doença. A forma em que o estresse oxidativo promove o desenvolvimento da doença é ilustrado com revisão da literatura em torno de seis doenças crônicas, a saber: câncer, doença cardiovascular, catarata, degeneração macular, doenças neurodegenerativas do sistema central e diabete melito.

Câncer: Em relação ao câncer, existem muitos registros da área de epidemiologia mostrando que o consumo abundante de frutas e verduras está ligado a um menor risco de vários tipos de câncer comum, como os de pulmão, cavidade oral, faringe, laringe e do colo uterino. Entretanto, é escassa a literatura que relata a ingestão de um ou outro nutriente específico como estando relacionada à incidência do câncer.

Doença cardiovascular (DCV): Dentre todas as doenças em que o excesso de estresse oxidativo tenha sido implicado, as DCV são as que contam com maiores evidências. A oxidação das lipoproteínas de baixa densidade parece ser o passo inicial para a ateroesclerose coronariana. Contudo, faltam estudos publicados que substanciem a noção de que consumindo mais vitamina $C$, vitamina $E$ ou $\beta$-caroteno (ou outros carotenóides) se reduza o risco de DCV.

Catarata: Os níveis séricos de vitamina C, vitamina E e carotenóides têm sido inversamente correlacionados com o risco de catarata. Elevação das concentrações séricas desses nutrientes através da suplementação, além do considerado normal, entretanto não demonstrou qualquer amenização da doença.

Degeneração macular relacionada com a idade (AMD): Estudos epidemiológicos mostram correlação entre o consumo de frutas e verduras ricas em carotenóides, particularmente em luteína e zeaxantina. Biologicamente, a função é possível, por quanto esses carotenóides se concentram e pigmentam a região da fovea centralis na retina. Embora indivíduos fumantes também possuam baixos níveis desses carotenóides no sangue e sejam grupos de risco para contrair AMD, espera-se ainda encontrar uma relação causal antes de acolher a luteína e a zeaxantina como nutrientes que diminuem o risco de AMD.

Doenças neurodegenerativas centrais: Existem evidências crescentes de que as doenças de Alzheimer, Parkinson e a esclerose amiotrófica lateral sejam manifestações adversas de estados de estresse oxidativo. Pequenos estudos de intervenção têm mostrado melhoria sintomática em pacientes já acometidos por essas doenças, quando tratados com vitamina C ou vitamina E. Todavia, como em outros casos, esses resultados são preliminares e não constituem prova de que a sua utilidade possa ser estendida à população em geral.

Diabete melito: Embora algumas evidências sugiram que a modificação de certas proteínas séricas e de outros tecidos em pessoas com diabetes melitus seja o resultado do estresse oxidativo, o tratamento desses indivíduos com antioxidantes não mostra respostas consistentes, sugerindo não ser a presença de espécies carbonílicas reativas, e sim a inabilidade do organismo de metabolizar ou inativar as carbonilas reativas, o possível fator causal.

\section{Quais foram as outras alterações?}

Além do enfoque mais voltado para a diminuição do risco de doenças, o novo agrupamento dos nutrientes, a extensa discussão da literatura pertinente e a criação das RDI, características estas que foram gerais para todos os volumes da obra até o momento lançados, está a modificação dos valores de algumas das recomendações.

No capítulo 1 se define a terminologia do novo relatório, como DRI, RDA, EAR, Al e UL, também as faixas etárias e estágios da vida. No capítulo 2 se definem os antioxidantes alimentares e se tecem as relações entre as doenças crônicas e os estados de estresse oxidativo. Este capítulo traz um quadro que resume todos os médios e grandes estudos de intervenção com antioxidantes no mundo, alguns dos quais se encontram ainda em andamento.

Já o capítulo 3 apresenta os métodos utilizados. De interesse para alguns leitores podem ser os métodos empregados para extrapolação de dados, tanto de adultos para crianças, como de recém-nascidos para bebês de mais idade (de zero a 6 meses, para de 7 a 12 meses). Esses procedimentos podem ser condensados nas fórmulas seguintes:

$$
\begin{aligned}
& \text { De adultos para crianças: } \\
& E A R_{\text {criança }}=E A R_{\text {adulto }}(F),
\end{aligned}
$$

onde $F$ é um fator de correção ( 1 + fator de crescimento), ou seja, $\left(\text { PesO }_{\text {criança }} / \text { Peso adulto }\right)^{0,75}$.

De lactentes jovens para mais velhos:

$$
A I_{7-12 \text { mês }}=A I_{0-6 \text { mês }}(F) \text {, }
$$

onde $F$ é um fator análogo, ou seja,

$$
\left(\text { PesO }_{7-12 \text { mês }} / \text { PesO }_{0-6 \text { mês }}\right)^{0,75} \text {. }
$$


Tabela 2. DRI (Ingestão Alimentar de Referência) para indivíduos, segundo a Food and Nutrition Board e o Institute of Medicine, da Academia Nacional de Ciências dos EUA (reproduzida com autorização).

\begin{tabular}{|c|c|c|c|c|c|c|c|c|c|c|c|c|c|c|c|c|c|}
\hline $\begin{array}{l}\text { Estágio da } \\
\text { vida }\end{array}$ & $\begin{array}{l}\text { Cálcio } \\
(\mathrm{mg} / \mathrm{d})\end{array}$ & $\begin{array}{c}\begin{array}{c}\text { Fósforo } \\
(\mathrm{mg} / \mathrm{d})\end{array} \\
\end{array}$ & $\begin{array}{c}\text { Magnésio } \\
\text { (mg/d) }\end{array}$ & $\begin{array}{l}\text { Vitamina D } \\
(\mu \mathrm{g} / \mathrm{d})^{a, b}\end{array}$ & $\begin{array}{c}\text { Fluoreto } \\
(\mathrm{mg} / \mathrm{d})\end{array}$ & $\begin{array}{c}\text { Tiamina } \\
(\mathrm{mg} / \mathrm{d})\end{array}$ & $\begin{array}{c}\text { Riboflavina } \\
(\mathrm{mg} / \mathrm{d})\end{array}$ & $\begin{array}{r}\text { Niacina } \\
(\mathrm{mg} / \mathrm{d})^{c}\end{array}$ & $\begin{array}{c}\text { Vitamina }_{(\mathrm{mg} / \mathrm{d})} \\
\text { Vin }\end{array}$ & $\begin{array}{l}\text { Folato } \\
(\mu \mathrm{g} / \mathrm{d})^{d}\end{array}$ & $\begin{array}{c}\text { Vitamina } B_{12} \\
(\mu \mathrm{g} / \mathrm{d})\end{array}$ & $\begin{array}{c}\text { Pantotenato } \\
(\mathrm{mg} / \mathrm{d})\end{array}$ & $\begin{array}{c}\text { Biotina } \\
(\mu \mathrm{g} / \mathrm{d})\end{array}$ & $\begin{array}{c}\text { Colina }^{e} \\
(\mathrm{mg} / \mathrm{d})\end{array}$ & $\begin{array}{c}\text { Vitamina C } \\
(\mathrm{mg} / \mathrm{d})\end{array}$ & $\begin{array}{c}\text { Vitamina } \mathrm{E}^{f} \\
(\mathrm{mg} / \mathrm{d})\end{array}$ & $\begin{array}{c}\text { Selênio } \\
(\mu \mathrm{g} / \mathrm{d})\end{array}$ \\
\hline \multicolumn{18}{|l|}{ Bebês } \\
\hline $0-6 \mathrm{~m}$ & $210 *$ & $100 *$ & $30 *$ & $5^{*}$ & & $0,2 *$ & $0,3^{*}$ & $2 *$ & $0,1^{*}$ & $65^{*}$ & $0,4^{*}$ & $1,7 *$ & $5^{*}$ & $125^{*}$ & $40 *$ & $4 *$ & $15^{*}$ \\
\hline $7-12 \mathrm{~m}$ & $270 *$ & $275^{*}$ & $75^{*}$ & $5^{*}$ & $\begin{array}{r}0,01^{*} \\
0,5^{*}\end{array}$ & $0,3 *$ & $0,4^{*}$ & $4 *$ & $0,3^{*}$ & $80^{*}$ & $0,5^{*}$ & $1,8^{*}$ & $6^{*}$ & $150^{*}$ & $50 *$ & $6^{*}$ & $20 *$ \\
\hline \multicolumn{18}{|l|}{ Crianças } \\
\hline $1-3$ a & $500^{*}$ & 460 & 80 & $5^{*}$ & $0,7^{*}$ & 0,5 & 0,5 & 6 & 0,5 & 150 & 0,9 & $2^{*}$ & $8^{*}$ & $200 *$ & 15 & 6 & 20 \\
\hline $4-8 \mathrm{a}$ & $800 *$ & 500 & 130 & $5^{*}$ & $1^{*}$ & 0,6 & 0,6 & 8 & 0,6 & 200 & 1,2 & $3^{*}$ & $12 *$ & $250 *$ & 25 & 7 & 30 \\
\hline \multicolumn{18}{|l|}{ Homens } \\
\hline $9-13$ a & $1300^{*}$ & 1250 & 240 & $5^{*}$ & $2 *$ & 0,9 & 0,9 & 12 & 1,0 & 300 & 1,8 & $4^{*}$ & $20 *$ & $375^{*}$ & 45 & 11 & 40 \\
\hline $14-18 \mathrm{a}$ & $1300^{*}$ & 1250 & 410 & $5^{*}$ & $3 *$ & 1,2 & 1,3 & 16 & 1,3 & 400 & 2,4 & $5^{*}$ & $25 *$ & $550^{*}$ & 75 & 15 & 55 \\
\hline $19-30 \mathrm{a}$ & $1000^{*}$ & 700 & 400 & $5^{*}$ & $4^{*}$ & 1,2 & 1,3 & 16 & 1,3 & 400 & 2,4 & $5^{*}$ & $30 *$ & $550 *$ & 90 & 15 & 55 \\
\hline $31-50 \mathrm{a}$ & $1000 *$ & 700 & 420 & $5^{*}$ & $4 *$ & 1,2 & 1,3 & 16 & 1,3 & 400 & 2,4 & $5^{*}$ & $30 *$ & $550 *$ & 90 & 15 & 55 \\
\hline $51-70$ a & $1200 *$ & 700 & 420 & $10 *$ & $4 *$ & 1,2 & 1,3 & 16 & 1,7 & 400 & $2,4^{g}$ & $5 *$ & $30 *$ & $550 *$ & 90 & 15 & 55 \\
\hline$>70 a$ & $1200 *$ & 700 & 420 & $15^{*}$ & $4 *$ & 1,2 & 1,3 & 16 & 1,7 & 400 & $2,4^{g}$ & $5^{*}$ & $30 *$ & $550 *$ & 90 & 15 & 55 \\
\hline \multicolumn{18}{|c|}{ Mulheres } \\
\hline $9-13$ a & $1300 *$ & 1250 & 240 & $5^{*}$ & $2 *$ & 0,9 & 0,9 & 12 & 1,0 & 300 & 1,8 & $4^{*}$ & $20 *$ & $375^{*}$ & 45 & 11 & 40 \\
\hline $14-18 \mathrm{a}$ & $1300^{*}$ & 1250 & 360 & $5^{*}$ & $3 *$ & 1,0 & 1,0 & 14 & 1,2 & $400^{h}$ & 2,4 & $5^{*}$ & $25^{*}$ & $400^{*}$ & 65 & 15 & 55 \\
\hline $19-30 \mathrm{a}$ & $1000^{*}$ & 700 & 310 & $5^{*}$ & $3 *$ & 1,1 & 1,1 & 14 & 1,3 & $400^{h}$ & 2,4 & $5^{*}$ & $30 *$ & $425^{*}$ & 75 & 15 & 55 \\
\hline $31-50 \mathrm{a}$ & $1000 *$ & 700 & 320 & $5^{*}$ & $3 *$ & 1,1 & 1,1 & 14 & 1,3 & $400^{h}$ & 2,4 & $5^{*}$ & $30 *$ & $425^{*}$ & 75 & 15 & 55 \\
\hline $51-70$ a & $1200 *$ & 700 & 320 & $10 *$ & $3 *$ & 1,1 & 1,1 & 14 & 1,5 & 400 & $2,4^{g}$ & $5^{*}$ & $30 *$ & $425^{*}$ & 75 & 15 & 55 \\
\hline$>70 \mathrm{a}$ & $1200^{*}$ & 700 & 320 & $15^{*}$ & $3 *$ & 1,1 & 1,1 & 14 & 1,5 & 400 & $2,4^{g}$ & $5^{*}$ & $30 *$ & $425^{*}$ & 75 & 15 & 55 \\
\hline \multicolumn{18}{|l|}{ Gestantes } \\
\hline$\leq 18 \mathrm{a}$ & $1300^{*}$ & 1250 & 400 & $5^{*}$ & $3 *$ & 1,4 & 1,4 & 18 & 1,9 & $600^{i}$ & 2,6 & $6^{*}$ & $30 *$ & $450 *$ & 80 & 15 & 60 \\
\hline $19-30 \mathrm{a}$ & $1000^{*}$ & 700 & 350 & $5^{*}$ & $3 *$ & 1,4 & 1,4 & 18 & 1,9 & $600^{i}$ & 2,6 & $6^{*}$ & $30 *$ & $450 *$ & 85 & 15 & 60 \\
\hline $31-50 \mathrm{a}$ & $1000^{*}$ & 700 & 360 & $5^{*}$ & $3 *$ & 1,4 & 1,4 & 18 & 1,9 & $600^{i}$ & 2,6 & $6^{*}$ & $30 *$ & $450 *$ & 85 & 15 & 60 \\
\hline \multicolumn{18}{|c|}{ Lactantes } \\
\hline$\leq 18 \mathrm{a}$ & $1300^{*}$ & 1250 & 360 & $5^{*}$ & $3 *$ & 1,4 & 1,6 & 17 & 2,0 & 500 & 2,8 & $7^{*}$ & $35^{*}$ & $550 *$ & 115 & 19 & 70 \\
\hline $19-30$ a & $1000^{*}$ & 700 & 310 & $5^{*}$ & $3 *$ & 1,4 & 1,6 & 17 & 2,0 & 500 & 2,8 & $7 *$ & $35^{*}$ & $550 *$ & 120 & 19 & 70 \\
\hline $31-50 \mathrm{a}$ & $1000^{*}$ & 700 & 320 & $5^{*}$ & $3 *$ & 1,4 & 1,6 & 17 & 2,0 & 500 & 2,8 & $7 *$ & $35^{*}$ & $550 *$ & 120 & 19 & 70 \\
\hline
\end{tabular}

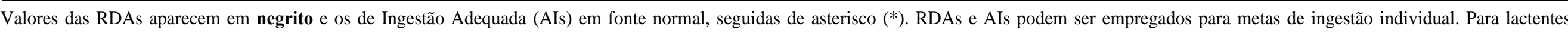

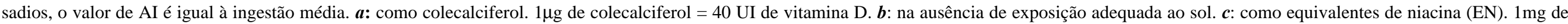

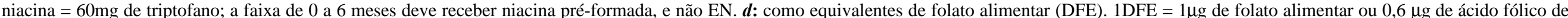

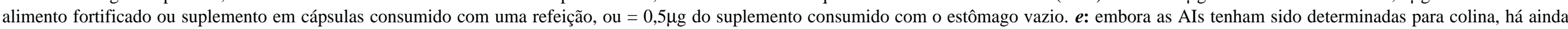

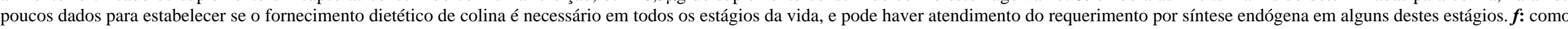

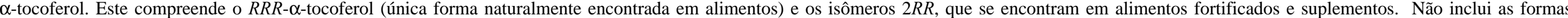

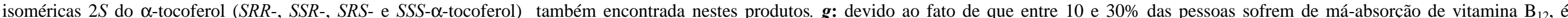

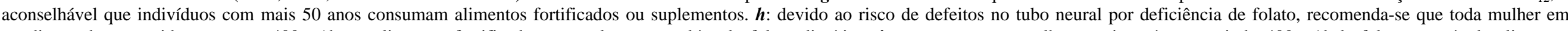

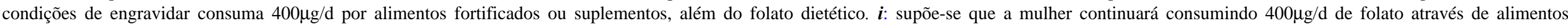

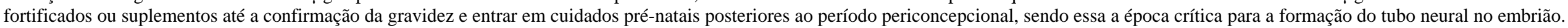


O capítulo 4, por sua vez, discute o procedimento para se chegar aos UL. Aqui se colocam os conceitos básicos de avaliação de risco, limiares, biodisponibilidade, interações nutriente-nutriente, fundamentos de toxicologia, Observable Adverse Effect Levels (OAEL) (Níveis de efeitos adversos observáveis), No Observable Adverse Effect Level (NOAEL) (Nível para o qual não há efeito adverso observável) e Low Observable Adverse Effect Level (LOAEL) (Nível para o qual há escasso efeito adverso observável).

Os capítulos 5, 6, 7 e 8 são os mais extensos e estão dedicados à revisão crítica da literatura da vitamina $\mathrm{C}$, vitamina $E$, selênio e os carotenóides, respectivamente. Cada nutriente em particular conta com um conjunto bibliográfico seletivo de experiências com humanos compreendidas entre 1988 e 1999, inclusive. Dessa forma, quase nenhuma referência bibliográfica citada na $10^{a}$ edição das RDA de 1989 aparece na 1a edição das RDI de 2000 e, portanto, o livro da 10 a edição não deve ser ainda descartado. De valor para pesquisadores é a possibilidade de observar as lacunas do estado atual do conhecimento e a priorização dos objetivos em novas pesquisas que foram apontados pelas comissões, especificamente para cada um desses nutrientes.

Com relação às alterações das recomendações numéricas, poderão ser vistas na tabela geral (Tabela 2) várias novidades:

1. Término da faixa etária de "crianças" na idade dos 8 anos, para iniciar a faixa de "homens" e "mulheres" com 9 a 13 anos. Também criação de mais duas faixas, para pessoas entre 51 e 70 e acima de 71 anos. De fato, entra em uso o termo 'estágio de vida', por ser mais abrangente do que 'faixa etária'.

2. Diminuição das recomendações de cálcio para crianças menores e aumento para jovens de até 18 anos. de fósforo.

3. Diminuição para a maioria das recomendações

4. Aumentos moderados para quase todos as recomendações de magnésio, assim como aumentos médios ( $50 \%$ ) para vitamina C e consideráveis (próximos de 100\%) para ácido fólico.

5. Diminuições discretas em todas as faixas e categorias para as vitaminas $\mathrm{B}_{1}, \mathrm{~B}_{2}$, niacina, $\mathrm{B}_{6}$ e vitamina $\mathrm{D}$.

6. Inclusão do fluoreto, ácido pantotênico, biotina e colina na tabela geral.

O Capítulo 9 refere-se ao uso correto das DRI, tanto na avaliação como no planejamento de dietas. Para cada situação, a aplicação pode se dar no nível de indivíduos ou populações.

Por exemplo, a EAR e o UL podem servir como componentes dos gabaritos de avaliação do consumo de um cliente de uma clínica, enquanto que a RDA e a AI serão componentes úteis no planejamento de uma dieta melhorada para o mesmo indivíduo. Da mesma forma, a
EAR e o UL podem ser usados na avaliação da adequação em nutrientes de uma população objeto de um inquérito, ou para planejar dietas para um determinado grupo, especialmente indivíduos institucionalizados.

O relatório traz, no capítulo 10, uma 'agenda de pesquisa que deveria nortear o desenvolvimento de futuros trabalhos na área, visando a sua utilização imediata nos próximos relatórios da Comissão. Estes itens podem ser brevemente resumidos em:

- Identificar as lacunas no conhecimento.

- Ponderar os dados para identificar quaisquer grandes inconsistências entre a ingestão e a 'estimativa dos requerimentos médios' (EAR). Tentar encontrar explicação para tais inconsistências. A explicação evitará recaídas futuras no mesmo problema.

- Levar em consideração mecanismos que protejam aqueles indivíduos com vulnerabilidade extrema bem conhecida, seja por pré-condição genética ou doença adquirida.

- Ponderar as alternativas e estabelecer as prioridades após consultar especialistas.

Seguindo este roteiro, quatro áreas foram identificadas como da maior importância: 1) requerimentos de nutrientes, 2) problemas metodológicos relacionados à avaliação da ingestão de nutrientes e à avaliação da adequação da ingestão, 3) relações entre a ingestão de um nutriente e a saúde pública, e 4) efeitos adversos de nutrientes.

Finalmente, o livro apresenta uma série de quadros nos apêndices, dentre os que se destaca a tabela atualizada das recomendações numéricas para cálcio, fósforo, magnésio, vitamina $D$, fluoreto, tiamina, riboflavina, niacina, vitamina $B_{6}$, folato, vitamina $B_{12}$, ácido pantotênico, biotina, colina, além de vitamina $C$, vitamina $E$ e selênio. Outro quadro que chama a atenção é do das médias e distribuição dos percentis de consumo (ingestão) de antioxidantes dos Estados Unidos e National Health and Nutrition Examination Survey III, 1988-1994 (NHANES III) e Continuing Survey of Food Intake by Individuals, 1994-1996 (CSFII).

\section{AGRADECIMENTO}

Os autores agradecem o apoio do Projeto Carotenóides, do Programa de Núcleos de Excelência (PRONEX)-Financiadora de Estudos e Projetos (FINEP).

\section{REFERÊNCIAS BIBLIOGRÁFICAS}

ATBC Cancer Prevention Study Group. The effect of vitamin E and beta-carotene on lung cancer and other cancers in male smokers. New England Journal of Medicine, Cambridge, v. 330, p.1029-1035, 1994. 
BLOT, W.J., LI, J-Y., TAYLOR, P.R. et al. Nutrition intervention trials in Linxian, China: Supplementation with specific vitamin/mineral combinations, cancer incidence and disease-specific mortality in the general population. I Nat Cancer Inst, Bethesda, v.85, p.1483-1492, 1993.

KALLNER, A., HARTMANN, D., HORNING, D. On the requirements of ascorbic acid in man: steady-state turnover and body pool in smokers. American Journal of Clinical Nutrition, Bethesda, v.34, p.1347-1355, 1981.

OMENN, G.S., GOODMAN, G.E., THORNQUIST, M.D. et al. Risk factors for lung cancer and for intervention effects in CARET, the Beta-Carotene and retinol Efficacy Trial. J Nat Cancer Inst, Bethesda, v.88, p.1550-1559, 1996.
US NATIONAL ACADEMY OF SCIENCES (USA). Dietary Reference Intakes for vitamin $C$, vitamin $E$, selenium and carotenoids. Washington DC : National Academy Press, 2000, 506p. (A report on the antioxidants and related compounds. Subcommittees on Upper Reference Intake Levels of Nutrients and Interpretation of Uses of Dietary Reference Intakes and the Standing Committee on the Scientific Evaluation of Dietary Reference Intakes. Food and Nutrition Board).

Recebido para publicação em 13 de novembro de 2000 e aceito em 16 de janeiro de 2001. 